\title{
Halloween Storm Simulations with the Space Weather Modeling Framework
}

\author{
Tamas I. Gombosi, Gábor Tóth, Igor V. Sokolov, Ward B. Manchester, \\ Aaron J. Ridley, Ilia I. Roussev, Darren L. De Zeeuw, Kenneth C. Hansen, \\ Kenneth G. Powell, and Quentin F. Stout \\ Center for Space Environment Modeling, The University of Michigan, Ann Arbor, MI 48109, USA
}

In late October and early November 2003 a series of some of the most powerful solar eruptions ever registered shook the heliosphere. These "Halloween storms" damaged 28 satellites, knocking two out of commission, diverted airplane routes and caused power failures in Sweden, among other problems. Long-distance radio communications were disrupted because of the effects on the ionosphere, and northern lights (aurora borealis) were seen as far south as Florida. This paper presents preliminary results of our simulations of the largest solar storm period in recent history, the Halloween storms. These simulation were made possible by the newly developed Space Weather Modeling Framework and by the computing power of NASA's Columbia system.

\section{Introduction}

Satellites, global positioning systems, high frequency communications, oil pipelines, and electricity have all become facts of life, however they all rely on technologies that can be negatively affected by conditions in the near-Earth space environment. In recognition of this, the National Space Weather Program (NSWP) was initiated to mitigate the adverse effects of space weather. The NSWP is a multiagency federal research program with NASA, NSF, NOAA, DoD and DoE each playing central roles. The ultimate goal is to achieve timely, accurate, and reliable space environment observations, specifications, and forecasts. Thus, understanding, modeling, and predicting the space environment around the Earth (Geospace) remains a critical need for our society.

The Sun-Earth system is a complex natural system of many different, interconnecting elements. The solar wind transfers significant mass, momentum and energy to the magnetosphere, ionosphere, and upper atmosphere, and dramatically affects the physical processes in each of these physical domains.

The Center for Space Environment Modeling (CSEM) at the University of Michigan and its collaborators have recently built a Space Weather Modeling Framework (SWMF). The SWMF is designed to couple the models of the various physics domains in a flexible yet efficient manner, which makes the prediction of space weather feasible on massively parallel computers. Each model has its own dependent variables, a mathematical model in the form of equations of evolution, and a numerical scheme with an appropriate grid structure and temporal discretization. The physics domains may overlap with each other or they can interact with each other through a boundary surface. The SWMF is able to incorporate models from the community and couple them with modest changes in the software of an individual model.

The new computational technologies incorporated into SWMF combined with the power of new supercomputing ssytems, such as Columbia, enable us to carry out realistic space weather simulations of the entire Sun-Earth system faster than real time. This paper presents preliminary results of our simulations of the largest solar storm period in recent history, the Halloween storms.

\section{Weather in Space}

"Space weather" has been used to refer to the conditions on the Sun and in the solar wind, magnetosphere, ionosphere, and thermosphere that can influence the performance and reliability of space-borne and groundbased technological systems or can endanger human life or health. 
The solar corona is so hot $\left(>10^{6} \mathrm{~K}\right)$ that in open magnetic field regions it undergoes a transonic expansion, filling all of interplanetary space with a supersonic magnetized plasma flowing radially outward from the Sun. As this flowing plasma, which is called the solar wind, passes the Earth, it interacts strongly with the geomagnetic field, severely compressing the field on the dayside of the Earth, and drawing it out into a long, comet-like tail on the nightside. The confined region of geomagnetic field is called the Earth's magnetosphere.

Significant temporal variations of solar wind speed at the orbit of Earth occur due to the rotation of solar wind structures. Such variations can also be produced by the transient ejection of mass and magnetic field from the solar corona (coronal mass ejections or CMEs). Indeed, the most severe storms experienced in the Earth's space environment are driven by exceptionally fast CMEs that exhibit a strong southward magnetic field component throughout a significant fraction of their volume. These very fast CMEs, which are ejected from the corona at speeds of more than $1000 \mathrm{~km} / \mathrm{s}$, also drive strong hydromagnetic shocks. These shocks are efficient producers of energetic particles. Of course, a very fast CME is only effective in producing a severe geomagnetic storm when it is directed towards the Earth, and this fact presents a problem for those attempting to give forewarning of such storms.

The solar wind not only confines the terrestrial magnetic field within the magnetospheric cavity, but it also transfers significant mass, momentum, and energy to the magnetosphere, as well as to the ionosphere and upper atmosphere. One dramatic consequence of this interaction between the solar wind and the magnetosphere is the production of a variety of complex electric current systems, ranging from a sheet of current flowing on the boundary between the solar wind and magnetosphere, to an enormous ring of current flowing around the Earth in the inner magnetosphere, to currents flowing throughout the ionosphere and connecting along magnetic field lines to magnetospheric currents systems. Another result of the solar-wind/magnetosphere interaction is the production of populations of very energetic particles that are stored in the magnetosphere and

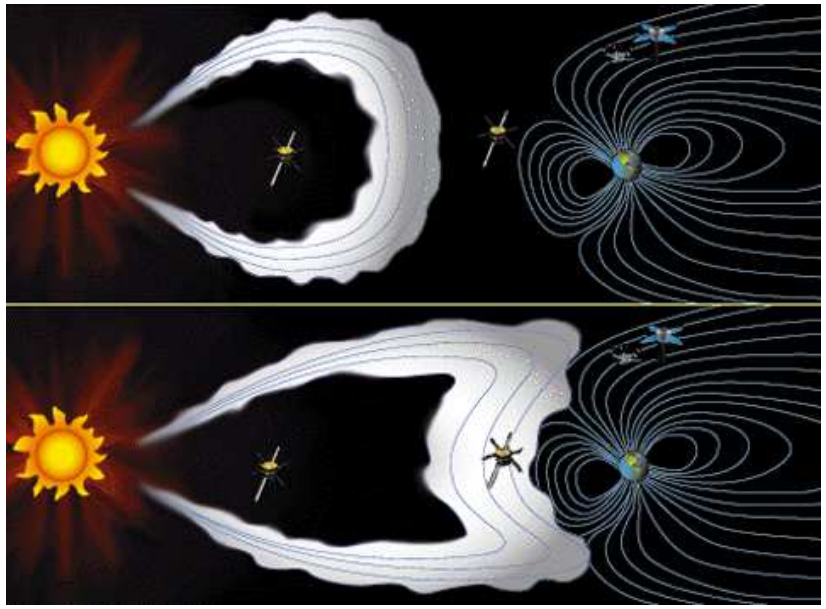

Figure 1. Schematic representation of the interaction of the magnetosphere with an expanding magnetic cloud. precipitated into the upper atmosphere. Both the electric currents and the energetic particles can have severe consequences for a number of human activities being carried out in various locations, all the way from the ground to space. It is the variation over time of these electric current systems and energetic particle populations in the geospace environment that modulates the consequences for human activities, and that is consequently the source of what we refer to as space weather.

A CME and its interaction with the magnetosphere is illustrated in Figure 1. The magnetic cloud generated by the CME approaches the magnetosphere in the top frame. In the bottom frame the cloud interacts with the magnetosphere and generates stronger magnetospheric current systems and larger, more energetic magnetospheric particle populations - a phenomenon which is called a geomagnetic storm. During magnetic storms the magnetospheric topology is significantly modified and and large transients are generated. As solar activity increases, the frequency of CMEs is substantially increased, and the "severity of space weather" is concomitantly increased.

\section{The Halloween Storms}

A solar flare is a sudden "flash" in the Sun's upper atmosphere at or near a sunspot. In late October and early November 2003 a series of some of the most powerful solar eruptions ever registered shook the heliosphere (see Figure 2). Dubbed "the Halloween storms," the blasts damaged 28 satellites, knocking two out of commission, diverted airplane routes and caused power failures in Sweden, among other problems. Long-distance radio communications were disrupted because of the effects on the ionosphere, and northern lights (aurora borealis) were seen as far south as Florida. The effects of the Halloween storms didn't stop at Earth. They went beyond to burn out the radiation monitor aboard the Global Surveyor spacecraft orbiting Mars. That instrument had been tracking the radiation future explorers might encounter on trips to the Red Planet. And beyond Mars near the planet Saturn, the Cassini spacecraft measured the intense energy from 
the Sun. Months later, the storms reached beyond Pluto's orbit to the edge of the Solar System, washing over the Voyager spacecraft.

Flares are classified by the order of magnitude of the peak burst intensity measured at the Earth in the 1 to $8 \AA$ band. The most powerful flashes are called "X-class" flares and they are characterized by the factor their peak intensity exceed the level of $10^{-4}$ Watts $/ \mathrm{m}^{2}$. The Halloween storms followed a two-month quiet period with the emergence of active region 10484 producing an X1.1 flare on October 19. During the next five weeks, two additional active regions, 10486 and 10488, produced some of the most intense flare activity and associated geomagnetic storms during Solar Cycle 23. While each of these regions was remarkable in size and magnetic complexity, Region 10486 was by far the most significant. With a size exceeding 2600 millionths of the solar disk (over 13 times the size of Earth), region 10486 was the largest sunspot group observed since November 1990. It maintained its extreme size, complex magnetic structure, and thus, great eruption potential, during its entire transit across the visible solar disk. Twelve of the seventeen major eruptions that occurred during this period came from Region 10486. Of the twelve major events, three stand out as defining events: the X17 on October 28, X10 on October 29, and X28 on November 4, 2003.

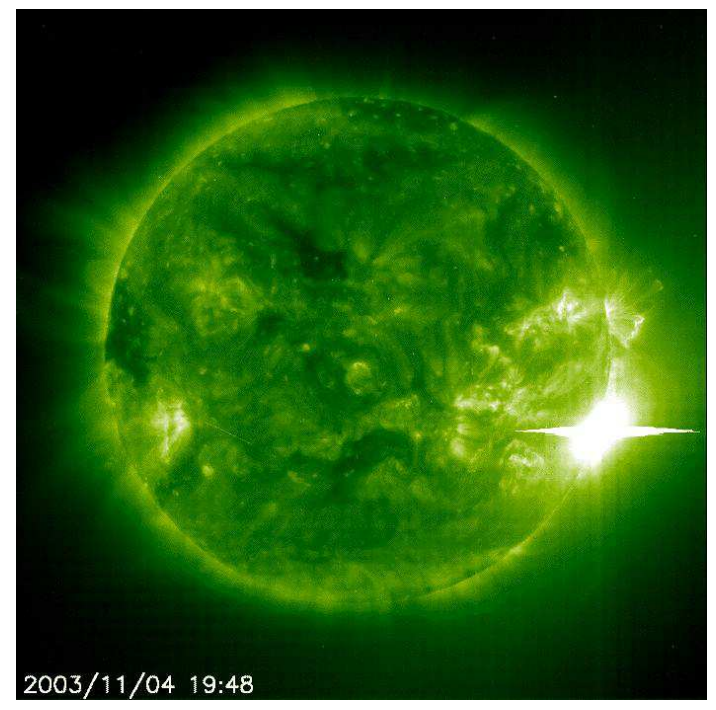

Figure 2. The solar flare of November 4 was the largest X-ray flare ever recorded. (SOHO/EIT ESA \& NASA)

\section{The Space Weather Modeling Framework}

In a number of fields in which computer-based modeling of complex, multi-scale, multi-physics problems plays an important role, software frameworks are being developed. The term software framework lacks a unique definition: some groups refer to a collection of models that interact through a coupling mechanism, however simple or intricate the coupling, as a software framework; other groups refer to the coupling software itself, independent of the models that it can couple, as a software framework.

The $\mathrm{SWMF}^{1,2}$ falls under the coupling-plus-models definition of a framework. It aims at providing a flexible and extensible software architecture for multi-component physics-based space weather simulations, as well as for various space physics applications. The main design goals are: (i) incorporate computational physics modules with only modest modification; (ii) achieve good parallel performance in the coupling of the physics components; and (iii) allow physics components to interact with the SWMF as efficiently as possible.

The SWMF includes a superstructure layer that drives the coupled-model application, and an infrastructure layer that provides utilities and data structures for model developers. For each model a wrapper needs to be written that can make use of the infrastructure layer. The individual models are coupled together with the efficient SWMF parallel coupling toolkit.

The SWMF is a fully functional and documented framework that provides a high-performance computa-

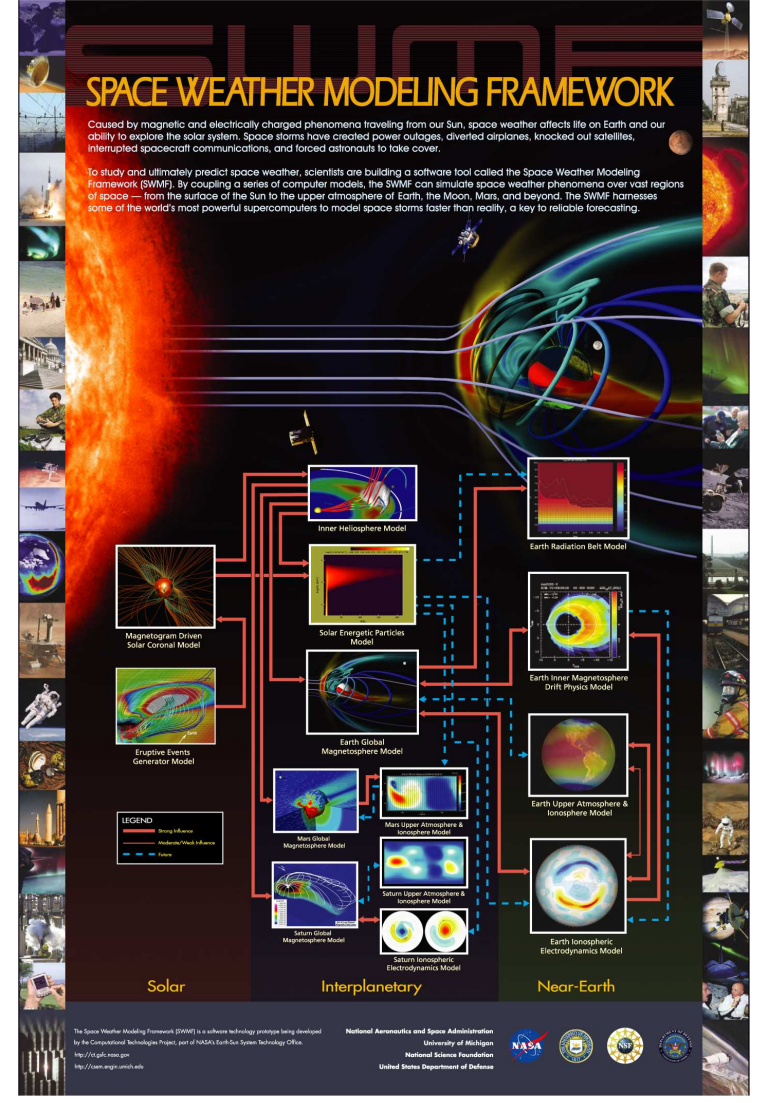

Figure 3. The SWMF poster (courtesy NASA). 
tional capability to simulate the physics from the low solar corona to the upper atmosphere of the Earth.,2 The 122-page SWMF User Manual explains the use of the SWMF with examples. It includes full documentation of the input parameters, testing procedures and code maintenance (http://csem.engin.umich.edu/SWMF). One of the most important features of the SWMF is that it can incorporate different computational physics modules to model different domains of Sun-Earth system. Each module for a particular domain can be replaced with alternatives, and one can use only a subset of the modules if desired.

\section{A. SWMF Modules.}

The SWMF integrates interoperating models of physics domains, ranging from the surface of the Sun to the upper atmosphere of the Earth. Each region is described by a world-class model, and the coupled models result in a self-consistent whole.

Presently the following modules are in SWMF:

\section{Solar Corona $(S C)$}

The Solar Corona domain extends from the surface of the Sun to approximately $24 R_{\odot}$. The physics of this domain is well approximated with the equations of magnetohydrodynamics, however, additional source terms are required to take into account the heating and acceleration of the solar wind..$^{3,4}$

The inner boundary of the SC component is driven by the density, pressure, velocity and magnetic field defined just above the photosphere. The magnetic field may be obtained from magnetograms, or a simple dipole may be assumed. The boundary conditions for the temperature and mass density at the Sun may vary with longitude and latitude to achieve the most realistic solar wind near the Sun and at 1AU. The velocity components at the inner boundary should maintain line-tying of the magnetic field. The flow at the outer boundary is usually superfast (faster than the fast magnetosonic speed of the plasma), so no information is propagating inward. Sometimes, however, when a coronal mass ejection (CME) passes the boundary, the solar wind speed may become subfast for short periods of time. During such periods, the SC component needs to receive the outer boundary condition from the Inner Heliosphere.

\section{Eruptive Event Generator (EE)}

The EE domain is embedded in the Solar Corona, and it is restricted to the region responsible for the eruptive event, or in other words, a coronal mass ejection (CME). The EE component can be represented as a boundary condition for the SC component, or it can be a (non-linear) perturbation of the SC solution. In short, the EE component interacts with the SC component only. Due to the multitude of possibilities, the EE component is integrated into the SC component in the current implementation of the SWMF. Multiple $\mathrm{EE}$ versions are possible, but all the EE versions belong to one $\mathrm{SC}$ version only.

\section{Inner Heliosphere (IH)}

The IH domain extends from around $20 R_{\odot}$ all the way to the planet. It does not have to cover a spherical region, it may be rectangular and asymmetric with respect to the center of the Sun. The physics of this domain is well approximated with the equations of ideal MHD. The IH component is usually in an inertial frame.

The inner boundary conditions of the IH component are obtained from the SC component or measurements. The flow at the outer boundary of the IH component is always superfast (the interaction with the interstellar medium is outside of the IH). The Inner Heliosphere provides the same information to the SP component as the Solar Corona. The IH component also provides the outer boundaries for the SC component when the flow at the outer boundary of SC is not superfast. Finally the Inner Heliosphere provides the upstream boundary conditions for the Global Magnetosphere (GM). The IH and GM domains overlap: the upstream boundary of GM is typically at about $30 R_{e}$ from the Earth towards the Sun, which is inside the IH domain. 


\section{Solar Energetic Particles (SP)}

The SP domain consists of one or more one dimensional field lines which are assumed to advect with the plasma. The physics of this domain is responsible for the acceleration of the solar energetic particles along the field lines. There are various mathematical models that approximate this physical system. They include the effects of acceleration and spatial diffusion, and can be averaged ${ }^{5}$ or non-averaged ${ }^{6}$ with respect to pitch angle.

The geometry of the field line and the plasma parameters along the field line are obtained from the SC and IH components. The boundary conditions can be zero particle flux at the ends of the field line(s). The SP component does not currently provide information to other components.

\section{Global Magnetosphere (GM)}

The GM domain contains the bow shock, magnetopause and magnetotail of the planet. The GM domain typically extends to about $30 R_{e}$ on the day side, hundreds of $R_{e}$ on the night side, and 50 to $100 R_{e}$ in the directions orthogonal to the Sun-Earth line. The physics of this domain is well approximated with the resistive MHD equations except near the planet, where it overlaps with the Inner Magnetosphere (IM).

The upstream boundary conditions are obtained from the IH component or from satellite measurements. At the other outer boundaries one can usually assume zero gradient for the plasma variables since these boundaries are far enough from the planet to have no significant effect on the dynamics near the planet. The inner boundary of the Global Magnetosphere is at some distance from the center of the planet, usually at 1 to 3 planet radii. The inner boundary conditions are partially determined by the Ionosphere Electrodynamics, which provides the electric potential at the inner boundary of the GM. The potential is used to calculate the electric field and the corresponding plasma velocities, which are used as the inner boundary condition for the GM. The GM component also receives pressure and possibly density corrections from the Inner Magnetosphere along the closed magnetic field lines (field lines connected to the planet at both ends). These are used to 'nudge' the MHD solution towards the more accurate inner magnetosphere values. ${ }^{7}$

The GM component provides the field aligned currents to the IE component. These currents are mapped from the GM down to the ionosphere along the magnetic field lines. The Global Magnetosphere provides the Inner Magnetosphere with the field line volume, average density and pressure along closed field lines. Depending on the needs of the IM component, the GM could also provide the geometry of the closed field lines and the distribution of plasma parameters along field lines.

\section{Inner Magnetosphere (IM)}

The IM domain consists of the closed field line region around the planet. This component solves equations describing the motion of the keV-energy ions and electrons. Kinetic effects are important for these particles, and the physics of this domain can be approximated in different manners. The Rice Convection Model ${ }^{8}$ uses a two dimensional bounce averaged description of a multi-energy plasma with gradient and curvature drift.

The Inner Magnetosphere obtains the geometrical and plasma information about the closed field lines from the Global Magnetosphere. It also obtains the electric potential solution and the radial current from the Ionosphere Electrodynamics. The IM component provides the density and pressure corrections along the closed field lines to the GM component.

\section{Radiation Belt (RB)}

The RB spatial domain is coincident with that of the Inner Magnetosphere component. This component solves equations for the relativistic electron distribution near the Earth which are responsible for some of the most detrimental space weather effects. Gradient and curvature drift dominate the motion of these particles around the Earth, and the kinetic equation is sometimes drift-shell averaged as well as gyration and bounce averaged. Diffusion is the primary transport mechanism left in the equation. The Radiation Belt receives similar information from the Global Magnetosphere as does the Inner Magnetosphere. The RB component does not provide information to the other components. 


\section{Ionosphere Electrodynamics (IE)}

The IE domain is a two dimensional height-integrated spherical surface at a nominal ionospheric altitude (at around $110 \mathrm{~km}$ for the Earth). In the current version of the SWMF, the IE component is a potential solver, but there is nothing in the design that would exclude the incorporation of other types of IE models.

The Ionospheric Electrodynamics obtains the field aligned currents from the Global Magnetosphere and Upper Atmosphere, which is used to generate an auroral precipitation pattern. The UA component also provides IE with the Hall and Pedersen conductivities. In case the UA component is not used, the auroral pattern and the solar illumination are used to generate Hall and Pedersen conductances. The IE component provides the electric potential to the GM, IM and UA components. In addition, it provides the radial currents to the IM component and the particle precipitation to the UA component.

\section{Upper Atmosphere (UA)}

The UA domain includes the thermosphere and the ionosphere and it extends from around $90 \mathrm{~km}$ to about $600 \mathrm{~km}$ altitude for the Earth. The physics of the Upper Atmosphere is rather complicated. It can be approximated with the equations of multi-species hydrodynamics including viscosity, thermal conduction, chemical reactions, ion-neutral friction, coupling of the ions to the electric field, source terms due to solar radiation, etc.

The lower and upper boundaries of the UA domain are approximated with physically motivated boundary conditions. The Upper Atmosphere obtains the electric potential along the magnetic field lines and the particle precipitation from the Ionosphere Electrodynamics. The gradient of the potential provides the electric field which is used to drive the ion motion, while the auroral precipitation is used to calculate ionization rates. The UA component provides field aligned currents and the Hall and Pedersen conductivities to the IE component. The conductivities are calculated from the electron density and integrated along field lines.

\section{B. Control and Coupling.}

The models are coupled together by the framework including a control module that determines the overall time-stepping of the code, the parallel decomposition of the models, the initiation and termination of the model runs, and the saving of restart files of the models. This involves code that determines when the coupling should occur, how it happens, grid interpolation, message passing between different components, and synchronization of the model runs to allow for a physically meaningful coupling.

The SWMF uses a component architecture (Figure 4), with each component created from a physics module by making some minimal changes and by adding two relatively small units of code: (i) a wrapper, which provides the standard interface to control the physics module; and (ii) coupling interface, to perform the data exchange with other components.

Both the wrapper and the coupling interface are constructed from building blocks provided by the framework. The structure of a component and its interaction with the Control Module $(\mathrm{CON})$ and another component are illustrated in Figure 5 .

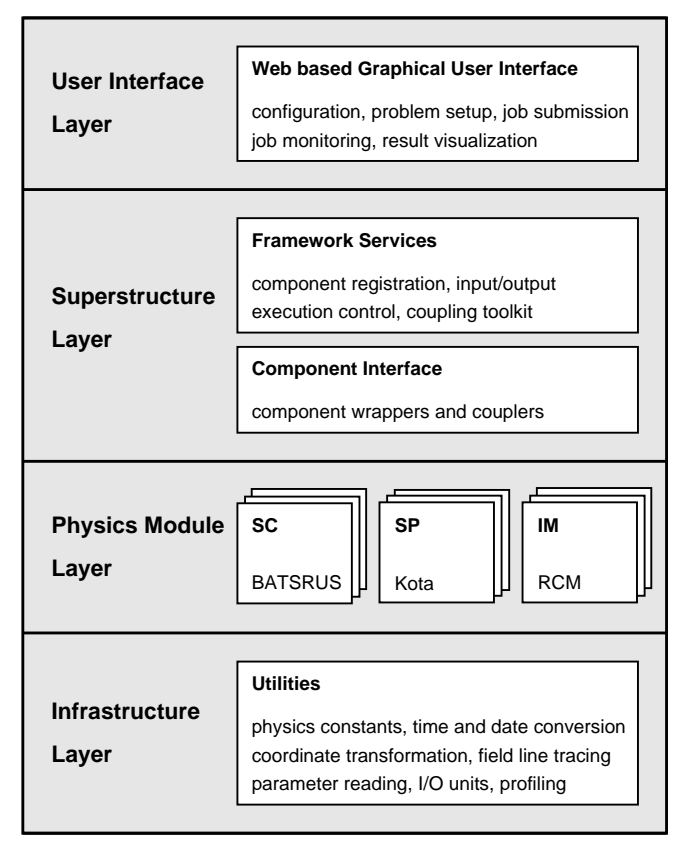

Figure 4. SWMF layers.

\section{Global MHD Model: BATS-R-US}

One of the modules within the SWMF is BATS-R-US, ${ }^{9-11}$ a flexible global MHD code that has the capability of modeling the Earth's magnetosphere, the solar corona, the inner and outer heliosphere, the magnetospheres of comets, Mars, Jupiter and Saturn, and other magnetized and unmagnetized bodies. 
The BATS-R-US code solves the governing equations of magnetohydrodynamics. Non-ideal MHD terms are included through appropriate source terms. The code uses a limited reconstruction that ensures second-order accuracy away from discontinuities, while simultaneously providing the stability that ensures non-oscillatory solutions. In addition, the code employs several approximate Riemann solvers. The resulting scheme solves for the hydrodynamic and electromagnetic effects in a tightly coupled manner, yielding a conservative scheme that works equally well across a range of several orders of magnitude in plasma $\beta$ (the ratio of plasma pressure to magnetic pressure).

\section{Data Structure and AMR.}

In BATS-R-US a relatively simple yet effective block-based adaptive mesh refinement (AMR) is used in conjunction with a finite-volume scheme. ${ }^{12}$ The computational cells are embedded in regular structured blocks of equal sized cells. The blocks are geometrically self-similar. Solution data associated with each block are stored in standard indexed array data structures. Computational grids are composed of many self-similar blocks. Adaptation is accomplished by the dividing and coarsening of appropriate solution blocks (see Figure 6). In regions requiring increased cell resolution, a "parent" block is refined by dividing itself into eight "children" or "offspring." In regions that are deemed over-resolved, the refinement process is reversed, and eight children are coarsened and coalesced into a single parent block. In this way, the cell resolution is changed by a factor of 2 .

\section{General Non-Cartesian Grid.}

We have recently implemented generalized curvilinear coordinates into BATS-R-US. Generalized coordinates provide a smooth mapping from a logically Cartesian grid to an arbitrary curvilinear grid, including spherical, cylindrical and toroidal grids. The generalized grids allow us to match non-planar boundary surfaces more accurately and it also allows the resolution to be concentrated in a continuous manner. Combined with the block-adaptive mesh refinement, the generalized coordinates provide an extremely flexible, efficient and accurate grid structure.

\section{Implicit Time-Stepping.}

The main limitation of most plasma codes is the explicit time stepping algorithm. Explicit time steps are limited by the
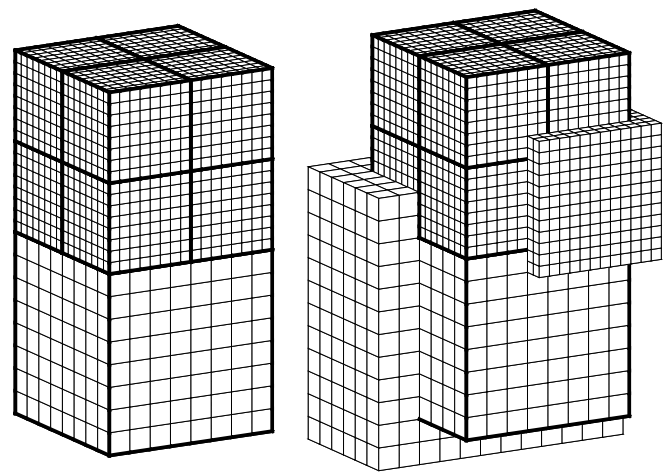

Figure 6. (left) Self-similar blocks used in BATS-R-US. (right) Double layer of ghost cells for both coarse and fine blocks.

Courant-Friedrichs-Lewy (CFL) condition, which essentially ensures that no information travels more than a cell size during a time step. This condition represents a non-linear penalty for highly resolved calculations, since finer grid resolution not only results in more computational cells, but also in smaller time steps.

We have implemented a fully implicit time stepping scheme especially designed for good parallel performance. ${ }^{13,14}$ We can also combine explicit and implicit time stepping. In global magnetospheric simulations, the combined explicit-implicit time stepping scheme improves the speed of the BATS-R-US code by a factor of 20 or more.

\section{Hail to Columbia}

The availability of NASA's Columbia system makes it poossible to carry out realistic simulations of superstorms in space. The large Halloween events certainly qualify for this category. Our experience shows that it takes about $1.6 \times 10^{8}$ state variables to resolve the main features of a superstorm as it is generated at 
the Sun, propagates through the inner heliosphere and interacts with geospace. A complete simulation takes about 30,000 processor hours on the Columbia system (using $>128$ processors). This is very large resource requirement if someone wants to use ensemble modeling techniques to estimate uncertainities. Unfortunately, our access to the Columbia system is somewhat limited due to the present resource allocation system.

The SWMF reaches faster than real-time performance. Figure 7 shows the ratio of simulation and CPU times on up to 256 processors of three supercomputers: the SGI Altix system at NASA Ames (Columbia), the SGI Origin 3800 at NASA Ames (Lomax) and the Compaq ES45 at NASA GSFC (Halem). On 256 CPU's of Columbia, which is the fastest (per CPU) of these supercomputers, the SWMF can run almost twice as fast as real time. We emphasize that this speed is achieved with reasonable spatial and temporal resolution in all science modules (the resolution is different in the different modules), and we used a total of 47 million state variables in the discretized physics domains).

\section{Halloween Storm Simulation}

We present preliminary simulation results for the Oc-

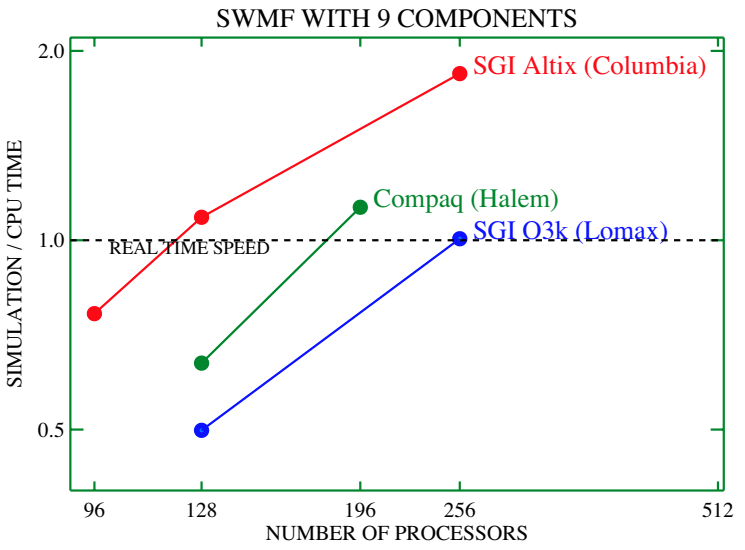

Figure 7. The ratio of simulation and CPU times on various supercomputers.

tober 28, 2003 Halloween storm, one of the most energetic CMEs observed, which was associated with an X17.2 flare. More detailed analysis of the event will be presented in follow-up publications.

\section{A. Sun-to-Earth}

The October 28 event was preceeded by several smaller CMEs, which significantly modified the ambient solar wind. To take into account the effect of previous CMEs we started the run from October 26, when a smaller CME was launched at around 06:00 UT from the same active region (AR10486).

We used the high resolution MDI synoptic maps centered around October 28 to set up the rotating initial solution of the $\mathrm{SC}$ and $\mathrm{IH}$ models. Using the block adaptive grid, BATS-R-US resolved the active region with cells as small as $3 \times 10^{-3} R_{\odot}$, while the total number of cells still remained around 2.5 million in the $\mathrm{SC}$ region. The $\mathrm{IH}$ grid contained more than 16 million cells ranging from $0.25 R_{\odot}$ to $4 R_{\odot}$. The vicinity of the Sun-Earth line is resolved with $0.25 R_{\odot}$ grid cells.

The eruptions were initiated with the EE by inserting a small flux rope (the size of the active region) based on the modified Titov and Démoulin ${ }^{16}$ model arching above the active region 10486 (Figure 8$)^{15}$. The location and orientation of the flux rope were chosen to match the observed photospheric magnetic field. The density in the loop was obtained from the size and estimated mass of the CMEs. The magnetic field strength of the flux ropes were set so that the resulting eruptions match LASCO

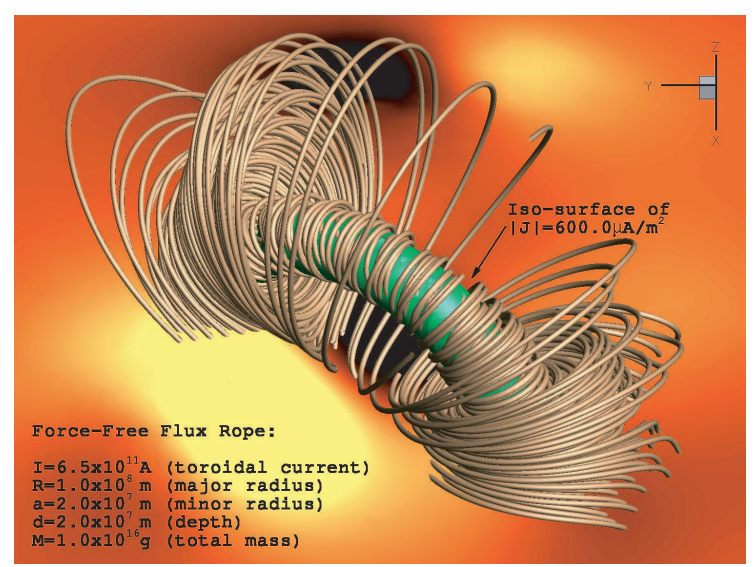

Figure 8. 3D view of the Roussev et al. ${ }^{15}$ flux rope superimposed to the background field of AR 10486. observations of the CME speeds at 20 solar radii $(1500$ and $2500 \mathrm{~km} / \mathrm{s}$ for the October 26 and October 28 events, respectively).

At the time of the eruption we switched to time accurate mode. After 20 minutes of the start of the time dependent simulation the leading shock of the first CME reaches a radial distance of $5.5 R_{\odot}$ at a speed slightly exceeding $2100 \mathrm{~km} / \mathrm{s}$. At this point the grid at the active region is coarsened by a factor of 4 , so that the simulation can be run more efficiently. The first CME reaches the SC/IH boundary at $20 R_{\odot}$ after about 1.7 hours and the speed of the leading shock is the observed $1500 \mathrm{~km} / \mathrm{s}$. The first CME is propagating in a direction about 30 degrees off from the Sun-Earth line, but the flanks of the shock reach the Earth 45 hours 
after the eruption at 3:00 UT Oct 28. The solar wind velocity at the Earth increases from about $350 \mathrm{~km} / \mathrm{s}$ to $550 \mathrm{~km} / \mathrm{s}$, which is a good approximation to the solar wind conditions preceding the arrival of the October 28 CME.

Before initiating the second CME, the active region is refined back to $3 \times 10^{-3} R_{\odot}$ resolution at 9:30 October 28 . The flux rope is made $50 \%$ larger and the magnetic field strength is increased so that the propagation speed of the CME reaches the $2500 \mathrm{~km} / \mathrm{s}$ measured at $20 R_{\odot}$. After $15 \mathrm{~min}-$ utes the second CME propagates to $9 R_{\odot}$ and the shock speed is around $3200 \mathrm{~km} / \mathrm{s}$. Again, the grid near the active region is coarsened to speed up the simulation. The shock reaches the SC/IH boundary at $20 R_{\odot}$ in less than an hour with a speed around $2800 \mathrm{~km} / \mathrm{s}$, and the second CME reaches the Earth 17.5 hours after the eruption at 3:00 October 29 (Figure 9).

The fastest plasma and strongest magnetic field of the second CME slightly missed the Earth. At Earth the velocity jumped from $450 \mathrm{~km} / \mathrm{s}$ to $1200 \mathrm{~km} / \mathrm{s}$ and the magnetic field components varied with 15 to $25 \mathrm{nT}$ amplitude. The difference in the arrival time and the strength of the CME is not unexpected given the limited information for the initial condition, the somewhat ad hoc initiation of the CME, and the various approximations in the physics.

In order to improve the agreement between the simulation and observations we moved Earth by $9^{\circ}$ along its orbit where the strongest part of the CME was passing through. Moving the Earth is an inexpensive way to do ensemble simulation. Now the shock speed reaches $1800 \mathrm{~km} / \mathrm{s}$ and the magnetic field varies in excess of $40 \mathrm{nT}$, which is in reasonable agreement with the observed values (see Figure 10). We also shifted the simulation time by 4.8 hours to match the observed arrival time of the shock.

It is very interesting to note that with these adjustments the simulation gives a very good agreement with the observed plasma parameters and the magnetic field magnitude. However, the simulated magnetic field components do not agree with the observations. This implies that the simulated magnetic field topology does not describe the complexity of the real CME. Realistically this is not surprising, since this is the first global CME simulation extending from the low corona to $1 \mathrm{AU}$ of a real event. Further research is needed to understand the physical or numerical reason for the discrepancy in the magnetic field components.

This simulation demonstrates several important advances in modeling heliospheric storms. First, the CMEs were launched from realistically sized active regions due to the very high local resolution made

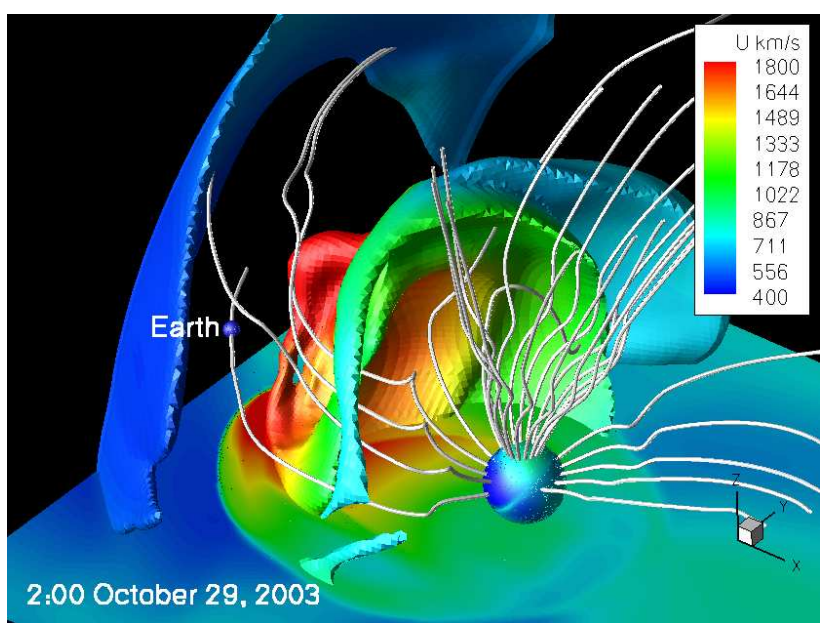

Figure 9. The heliosphere during the Halloween events of 2003. Disturbances are shown as isosurfaces where the density is increased by a factor of three over the ambient value. These surfaces are colored with the solar wind speed and they show the presence of shocks driven by the October 26 and October 28 CMEs. A plane ( $\left.\mathrm{z}=-40 R_{\odot}\right)$ and sphere (inner boundary of IH) are also shown colored to show flow speed. Gray lines illustrate the interplanetary magnetic field.
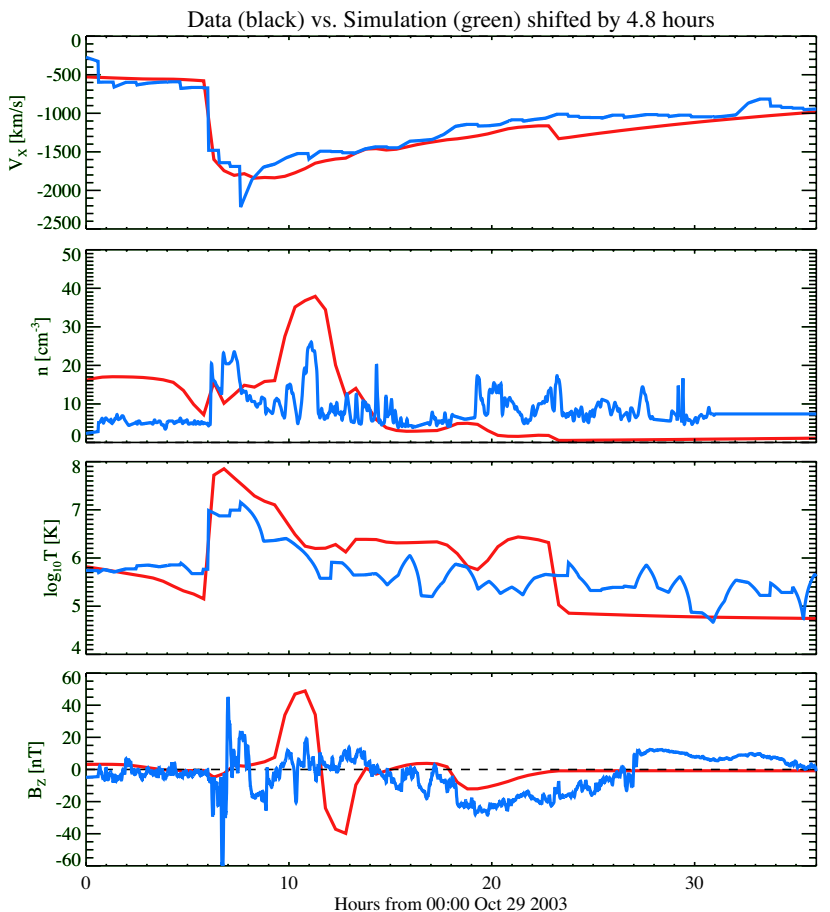

Figure 10. The solar wind velocity, density, temperature and $\mathrm{Bz}$ as measured by the $\mathrm{ACE}$ and Geotail satellites (blue) and the simulated values extracted at the slightly shifted Earth location (red). possible by the adaptive grid. Without adaptive grids one must use unrealistically large active regions even 
when the code is run at high resolution. Second, we demonstrated that simulating the "preconditioning" of the heliosphere by earlier CMEs is very important if one wants to compare in situ observations with simulation results. Preconditioning requires well resolved simulations over a long period and thus needs significant computational resources, even with very efficient codes. Third, we coupled the SC and IH modules for all MHD parameters without any "renormalization."

\section{B. Interaction with the Magnetosphere}

Two sets of magnetosphere simulations were carried out for the Halloween event. In the first case we used observations from upstream monitors to set the inflow boundary conditions. In the second case the global solar-heliospheric simulations described above were used as the upstream boundary conditions. In this simulation we used the GM, IM, UA and IE modules.

\section{Driving with Upstream Monitors}

In the first case the simulation was driven by observed solar wind and IMF values from the ACE and Geotail satellites.

Figure 11 shows a 3D representation of the magnetosphere at $08 \mathrm{UT}$ on October 29, 2003. It can be seen that a large ring current is present within the simulation, caused by a much larger ring current pressure than could be produced with MHD alone. This is due to the inclusion of a proper drift physics

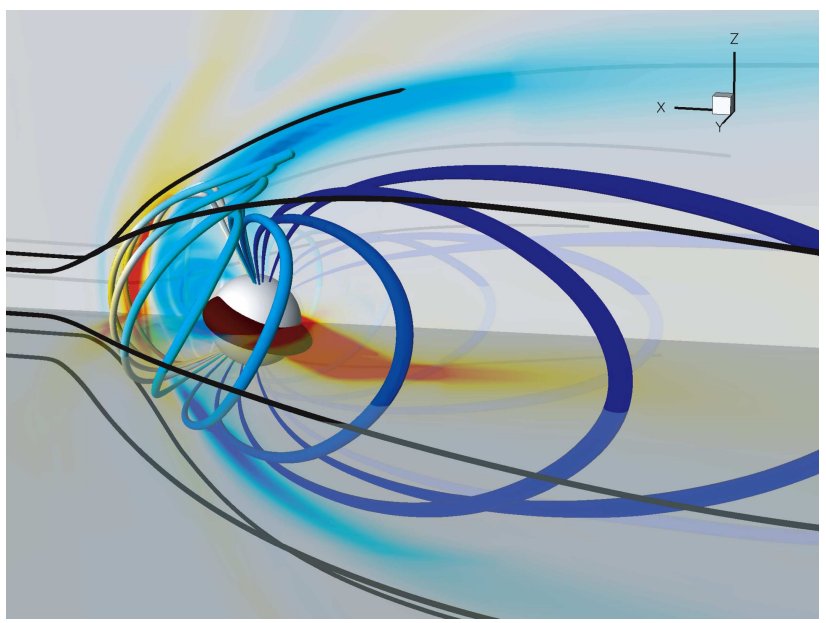

Figure 11. 3D structure of the magnetosphere during the main-phase of the October 29 storm. The colored cylinders show the last-closed magnetic field lines, while the black cylinders indicate the solar wind flow around the magnetosphere. The color contours on the planes are electric current density. The $100 \mathrm{nPa}$ pressure isosurface that intersects this boundary is also shown.

model (for the IM component we used the Rice Convection Model ${ }^{7}$ ). The large ring current pressure is essential for generating the proper Region 2 current system that connects the high-latitude ionosphere to the ring current. This coupling played an important role in driving the ionospheric convection pattern, and consequently, in controlling magnetospheric dynamics.

There are several planetary scale indices that characterize the overall dynamical state of the magnetosphere. While it is fully expected that numerical simulations cannot describe the detailed dynamics at any given point of the magnetosphere-ionosphere system (due to to limited resolution and the lack of detailed microphysics in the governing equations), a high quality model should be able to reproduce global indices with reasonable accuracy. Figure 12 shows a comparison between simulated and observed global indices. It shows the ionospheric cross polar cap potential (CPCP) predicted from the assimilative mapping of ionospheric electrodynamics technique (AMIE) and the SWMF, as well as the one-minute $D_{s t}$ from 54 ground-based magnetometers and the SWMF. These plots show that the SWMF does an extremely good job predicting these major indices even during the most disturbed periods.

Figure 13 shows comparisons between simulation results and data from three magnetospheric spacecraft during the storm. Cluster was in the dayside magnetosphere, the Polar spacecraft was near the Northern cusp, while GOES-10 was behind Earth at geosyncronous orbit. These plots show that we can reproduce the magnetic field at several locations around the near-Earth space environment. SWMF accurately models the motion of the magnetopause, as observed by the GOES-10 measurements of $B_{z}$ sign changes. We also note that the Wind satellite was located far
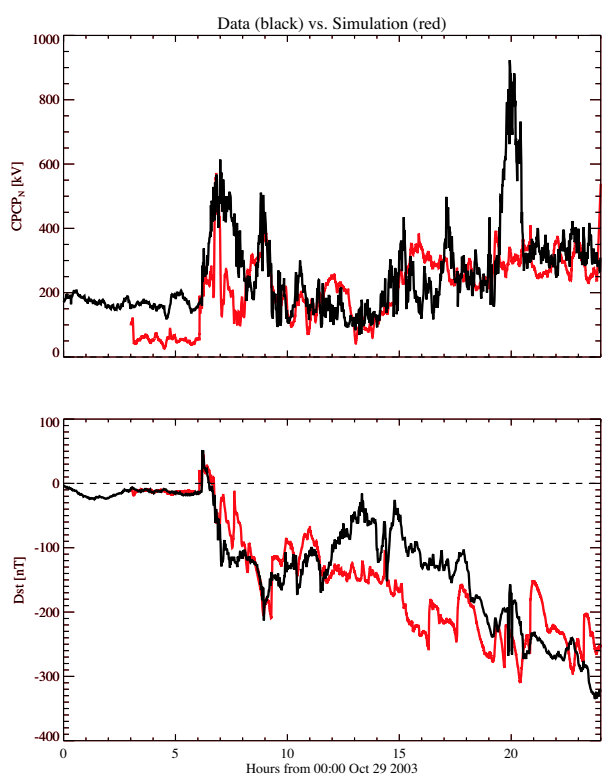

Figure 12. AMIE (black) and SWMF (red) results of the ionospheric cross polar cap potential (top) and $D_{s t}$ (bottom) for October 29, 2003. 
downtail (about $150 R_{e}$ ) during this period and our simulation is in excellent agreement with the Wind observations as well.
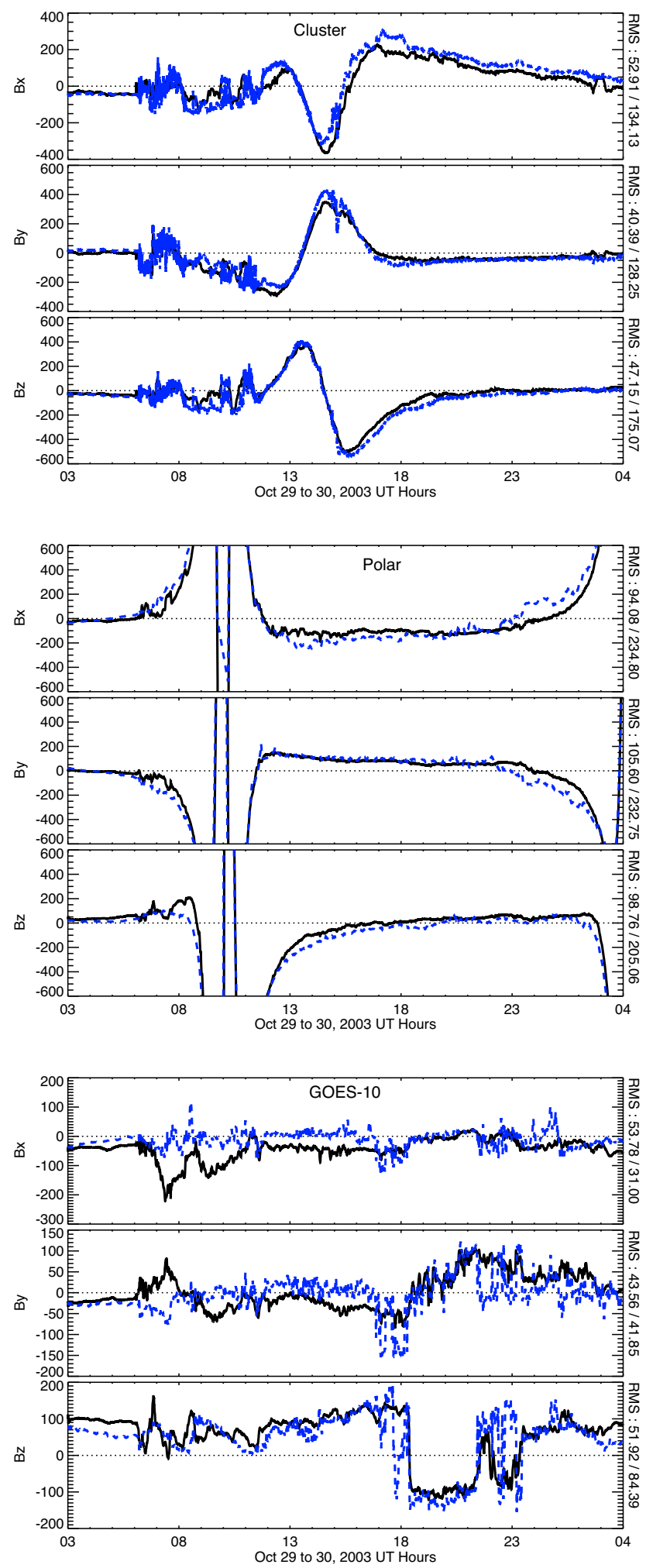

Figure 13. Comparison of the magnetic field measured by the Cluster, Polar and GOES-10 satellites (blue) with simulation results (black) for the October 29-30, 2003 storm.
Inspection of Figures 12 and 13 reveals several very interesting aspects of the simulation results. First of all, the global indices are reproduced amazingly well. The CPCP values obtained with the AIME assimilative method and produced by the simulation follow each other quite closely with the exception of a short period around 2000 UT on October 29. At this time the AIME potential reaches $800 \mathrm{kV}$, a value that is so high that the assimilative technique might lose validity. More importantly, the $D_{s t}$ index is directly obtained from ground magnetometer observations and it does not involve sophysticated data analysis. This index is also very well reproduced by the simulation, particularly during the first part of the magnetic storm.

Figure 13 shows that the simulation reproduces the observations in the dayside magnetosphere and in the cusp region extremely well. The agreement with Cluster and Polar is really good. In the closed field line region behind Earth the agreement is reasonable but there are several important features that are missed by the simulation. On the positive side, the simulation reproduces the $\mathrm{Bz}$ component, and thus captures the motion of the magnetopause. However, the transient feature around 2300 UT is missed by the simulation.

\section{Driving with Simulated CME}

We also carried out a simulation when the magnetosphere was driven by the simulated CME. These results are still being being analyzed, but preliminary interpretation indicates that the overall magnetospheric response to the superstorm is very reasonable. Global indices, such as CPCP and $D_{s t}$ generally follow the observations well, but the overall agreement is somewhat degraded as compared to the simulation driven by upstream observations (see Figure 12).

A very important aspect of the complete Sunto-Earth simulation was the resolution and speed. We note that this simulation involved over 160 million state variables. The active region on the Sun was resolved with grid sized of $1 / 340 R_{\odot}$, an unprecendent resolution in any global corona simulation. The heliosphere and the geospace was also resolved with good accuracy. On 200 CPUs of the Columbia system the simulation ran about 1.2 times slower than real time. Based on our scaling curves we estimate that we can run a well resolved SunEarth superstorm simulation at real time on 256 CPUs on Columbia, and about 1.5 faster than real time on 512 CPUs. 


\section{Conclusion}

A global simulation of an observed space superstorm has been carried out for the first time. The simulation was driven by observed synoptic solar magnetograms and with a sophysticated model of solar coronal mass ejections. The results are in general agreement with observetions.

A more detailed, upstream monitor driven magnetosphere simulation successfully reproduces simultaneous observations by a suit of satellites located in different regions of geospace. With the help of the newly developed Space Weather Modeling Framework we were able to reproduce the main characteristics of the dayside magnetosphere, the cusp, the region of closed magnetic field lines behind Earth, and the distant magnetotail. In addition, the global geomagnetic indices (like CPCP and $D_{s t}$ ) are also well reproduced by the simulation.

The simulation was made possible by the availability of NASA's Columbia system that allowed us to reach near-real time speed using 160 million state variables.

\section{Acknowledgments}

This work has been supported by NASA AISRP grant NNG04GP89G, NASA ESS CT cooperative agreement NCC5-614, and by DoD MURI grant F49620-01-1-0359. We gratefully acknowledge the supercomputing resources provided by NASA's Columbia system under award SMD1-Dec04-0099.

\section{References}

${ }^{1}$ Tóth, G., Volberg, O., Ridley, A. J., Gombosi, T. I., De Zeeuw, D. L., Hansen, K. C., Chesney, D. R., Stout, Q. F., Powell, K. G., Kane, K. J., and Oehmke, R., "A physics-based software framework for Sun-Earth connection modeling," Multiscale Coupling of Sun-Earth Processes, edited by A. T. Y. Lui, Y. Kamide, and G. Consolini, Elsevier, 2005, pp. 383-397.

${ }^{2}$ Tóth, G., Sokolov, I. V., Gombosi, T. I., D, R. C., Clauer, C. R., De Zeeuw, D. L., Hansen, K. C., Kane, K. J., Manchester, W. B., Oehmke, R. C., Powell, K. G., Ridley, A. J., Roussev, I. I., Stout, Q. F., Volberg, O., Wolf, R. A., Sazykin, S., Chan, A., and Yu, B., "Space Weather Modeling Framework: A new tool for the space science community," J. Geophys. Res., Vol. 110, No. A0, 2005, pp. doi:10.1029/2005JA011126, in press.

${ }^{3}$ Groth, C. P. T., De Zeeuw, D. L., Gombosi, T. I., and Powell, K. G., "Global 3D MHD simulation of a space weather event: CME formation, interplanetary propagation, and interaction with the magnetosphere," J. Geophys. Res., Vol. 105, No. A11, 2000, pp. 25,053-25,078.

${ }^{4}$ Usmanov, A. V., Goldstein, M. L., Besser, B. P., and Fritzer, J. M., "A global MHD solar wind model with WKB Alfvén waves: Comparison with Ulysses data," J. Geophys. Res., Vol. 105, 2000, pp. 12,675-12,695.

${ }^{5}$ Sokolov, I. V., Roussev, I. I., Gombosi, T. I., Lee, M. A., Kóta, J., Forbes, T. G., Manchester, W. B., and Sakai, J. I., "A new field line advection model for solar particle acceleration," Astrophys. J., Vol. 616, 2004, pp. L171-L174.

${ }^{6}$ Kóta, J. and Jokipii, J. R., "Transport of CIR accelerated particles," Proc. 26th Int. Cosmic Ray Conf., Vol. 6, AIP, 1999, p. 612.

${ }^{7}$ De Zeeuw, D., Sazykin, S., Wolf, R., Gombosi, T., Ridley, A., and Tóth, G., "Coupling of a Global MHD Code and an Inner Magnetosphere Model: Initial Results," J. Geophys. Res., Vol. 109, No. A12, 2004, pp. A12219, doi:10.1029/2003JA010366.

${ }^{8}$ Wolf, R. A., Harel, M., Spiro, R. W., Voigt, G., Reiff, P. H., and Chen, C. K., "Computer simulation of inner magnetospheric dynamics for the magnetic storm of July 29, 1977," J. Geophys. Res., Vol. 87, 1982, pp. 5949-5962.

${ }^{9}$ Powell, K. G., Roe, P. L., Linde, T. J., Gombosi, T. I., and De Zeeuw, D. L., "A Solution-Adaptive Upwind Scheme for Ideal Magnetohydrodynamics," J. Comput. Phys., Vol. 154, No. 2, Sept. 1999, pp. 284-309.

${ }^{10}$ Gombosi, T. I., Tóth, G., De Zeeuw, D. L., Hansen, K. C., Kabin, K., and Powell, K. G., "Semi-relativistic magnetohydrodynamics and physics-based convergence acceleration," J. Comput. Phys., Vol. 177, 2002, pp. 176-205.

${ }^{11}$ Gombosi, T. I., Powell, K. G., De Zeeuw, D. L., Clauer, C. R., Hansen, K. C., Manchester, W. B., Ridley, A. J., Roussev, I. I., Sokolov, I. V., and Tóth, G., "Solution Adaptive MHD for Space Plasmas: Sun-to-Earth Simulations," Computing in Science and Engineering, Vol. 6, No. 2, 2004, pp. 14-35.

${ }^{12}$ Stout, Q. F., De Zeeuw, D. L., Gombosi, T. I., Groth, C. P. T., Marshall, H. G., and Powell, K. G., "Adaptive blocks: A high-performance data structure," Proc. Supercomputing'97, 1997.

${ }^{13}$ Tóth, G., Keppens, R., and Botchev, M. A., "Implicit and semi-implicit schemes in the Versatile Advection Code: Numerical tests," Astron. Astrophys., Vol. 332, 1998, pp. 1159-1170.

${ }^{14}$ Tóth, G., De Zeeuw, D. L., Gombosi, T. I., and Powell, K. G., "A Parallel Explicit/Implicit Time Stepping Scheme on Block-Adaptive Grids," J. Comput. Phys., Vol. submitted, 2005.

${ }^{15}$ Roussev, I. I., Forbes, T. G., Gombosi, T. I., Sokolov, I. V., DeZeeuw, D. L., and Birn, J., "A Three-Dimensional Flux Rope Model for Coronal Mass Ejections Based on a Loss of Equilibrium," Astrophys. J., Vol. 588, 2003, pp. L45-L48.

${ }^{16}$ Titov, V. S. and Dèmoulin, P., "Basic topology of twisted magnetic configurations in solar flares," Astron. Astrophys., Vol. 351, 1999, pp. 701-720. 